\title{
THE COHOMOLOGY RINGS OF COMPLEMENTS OF SUBSPACE ARRANGEMENTS
}

\author{
MARK DE LONGUEVILLE AND CARSTEN A. SCHULTZ
}

\begin{abstract}
The ring structure of the integral cohomology of complements of real linear subspace arrangements is considered. While the additive structure of the cohomology is given in terms of the intersection poset and dimension function by a theorem of Goresky and MacPherson, we describe the multiplicative structure in terms of the intersection poset, the dimension function and orientations of the participating subspaces for the class of arrangements without pairs of intersections of codimension one. In particular, this yields a description of the integral cohomology ring of complex arrangements conjectured by Yuzvinsky. For general real arrangements a weaker result is obtained. The approach is geometric and the methods are elementary.
\end{abstract}

\section{Contents}

1. Introduction 1

2. Preliminaries 4

3. The Goresky-MacPherson isomorphism 5

4. Products 7

5. On $(\geq 2)$-arrangements 11

6. Counterexamples regarding the general case 14

7. A result regarding general arrangements 15

8. The homology of the link 18

References $\quad 20$

\section{INTRODUCTION}

The ring structure of the integral cohomology of complements of real linear subspace arrangements is the concern of this article. Since the additive structure of this cohomology is given by a result of Goresky and MacPherson [GM88], the task is to describe the multiplicative structure. In order to

2000 Mathematics Subject Classification. Primary: 52C35 Secondary: 05E25 14P25 $32 \mathrm{SS} 22$.

The first author was supported by the graduate school "Algorithmische Diskrete Mathematik". The graduate school "Algorithmische Diskrete Mathematik" is supported by the Deutsche Forschungsgemeinschaft, grant GRK 219/3. 
provide a context for our results we recall some previously achieved ones on the ring structure.

$\triangleright$ Using rational models De Concini and Procesi derived that the multiplicative structure of the rational cohomology in the case of complex arrangements is determined by combinatorial data: intersection lattice and dimension function [CP95]. Their techniques were applied by Yuzvinsky to give an explicit description for the rational cohomology ring for complex arrangements [Yuz98].

$\triangleright$ Arnol'd's investigation and conjectures on the cohomology ring of complex braid arrangements [Arn69] lead to the description of the cohomology ring of complex hyperplane arrangements by Brieskorn [Bri73] which was formulated and generalized in a combinatorial setting by Orlik and Solomon. Generalizing the Orlik-Solomon result on complex hyperplane arrangements [OS80] Feichtner and Ziegler obtained a presentation for the integral cohomology ring of the complement of a complex arrangement with geometric intersection lattice [FZ00] by extending combinatorial stratification methods from Björner and Ziegler [BZ92]. Independently, Yuzvinsky obtained this result as an application of his work on the rational cohomology rings of complex arrangements mentioned above [Yuz98], [Yuz99].

$\triangleright$ Ziegler gave a presentation for the integral cohomology ring of a real 2-arrangement [Zie93]. Applying this result he showed that intersection lattice and dimension function as combinatorial data do not suffice to determine the ring.

In this article we

$\triangleright$ describe the integral cohomology ring structure for general real arrangements up to an error term: Theorem 7.5,

$\triangleright$ determine the integral cohomology ring structure for $(\geq 2)$-arrangements, a class generalizing complex arrangements and real 2-arrangements: Theorem 5.2,

$\triangleright$ give a presentation for the integral cohomology ring of $(\geq 2)$-arrangements with geometric intersection lattice: Theorem 5.5.

Having Ziegler's result in mind we extend the combinatorial data by orientation information in the general case, i.e., all spaces in the arrangement are considered to have a specific orientation. Since all complex spaces possess a canonical orientation the orientation information becomes unnecessary in the case of complex arrangements. In this special case our result shows that, as Yuzvinsky conjectured, his description of the cohomology ring is also valid for integer coefficients [Yuz98], which was partially shown before for complex coordinate arrangements by one of the current authors [Lon00].

Apart from our new results this article unifies the results and simplifies the methods compared to those previously known: we employ elementary methods from combinatorics and topology only. 
Our results are based on the description of the homology of the link by Goresky and MacPherson [GM88] in the setting of the approach of Ziegler and Živaljević [ZŽ93] who provided a homotopy model of the link. In fact, there is a purely combinatorially defined ring structure on this homology given by the intersection lattice, the dimension function, and the orientation information. The main result is that this combinatorially defined ring structure coincides with the cohomology ring of the arrangement in case of a ( $\geq 2$ )-arrangement.

An important step in the proof is the insight that for $(\geq 2)$-arrangements the Goresky-MacPherson isomorphism is canonical. A crucial distinction in the description of the ring structure is given by a certain codimension condition: in all computations of cohomology rings of subspace arrangements that have been done in the past, cohomology classes multiply trivially as long as they do not satisfy such a condition (cf., e.g., [OS80], [BZ92], [FZ00], [Lon00]). This will also be true for the $(\geq 2)$-arrangements that we consider, however not for general real arrangements.

After a first version of this article was finished we learned about the recent work of Deligne, Goresky and MacPherson [DGM99], where similar questions are considered. By a sheaf theoretic approach using derived categories they obtain - among others - comparable results.

Organization of the paper. In Section 2 we first introduce notation. In particular, we define our notion of combinatorial data which is somewhat non-standard because of the fact that all spaces are considered to be oriented. Furthermore, the notion of generic points in our setting is introduced. In Section 3 we provide a version of the Goresky-MacPherson isomorphism, essentially relying on the work of Ziegler and Živaljević. The subsequent Section 4 is the heart of this article describing the combinatorial product on homology and relating it to the intersection product. The main theorem for the class of $(\geq 2)$-arrangements is now achieved in Section 5 as an application of the previous results. The section concludes with a presentation for the cohomology ring of a $(\geq 2)$-arrangement with geometric intersection lattice. Section 6 is an example section that demonstrates the importance of the restriction to $(\geq 2)$-arrangements. In Section 7 we will see what still can be said in the general case. Finally, a section explaining the correspondence of our approach in this paper to one starting with the homotopy model by Ziegler and Živaljević, which was our first approach, is appended. This section also draws the connection to the language of Yuzvinsky's article [Yuz98].

Acknowledgments. We would like to thank Elmar Vogt and Günter M. Ziegler for constant support and valuable advice during the time this work has been done. In particular, we want to thank Mark Goresky and Robert MacPherson for the critical reading of an earlier version of this paper, and for many helpful remarks. 


\section{Preliminaries}

Notation. Let $\mathcal{A}$ be a (linear) subspace arrangement in a finite-dimensional $\mathbb{R}$-vector space $W$, i.e., a finite family of linear subspaces of $W$. $\mathcal{A}$ may be empty, but must not contain $W$.

Let $u \subseteq v \subseteq W$ be a linear subspaces. By $\pi^{u}$ we denote the quotient map $W \rightarrow W / u$ and $\pi^{u, v}$ denotes the quotient map $\pi^{u, v}: W / u \rightarrow W / v$. We define $\mathcal{A}_{u}$ to be the subarrangement $\mathcal{A}_{u}:=\{z \in \mathcal{A}: u \subseteq z\}$ in $W$ and denote by $\mathcal{A} / u$ the arrangement in $W / u$ given by $\mathcal{A} / u:=\left\{\pi^{u}[z]: z \in \mathcal{A}_{u}\right\}$.

The objective of this paper is to relate topological and combinatorial data.

Topological data. Associated with the arrangement $\mathcal{A}$ is the link $L_{\mathcal{A}}=$ $S(W) \cap \bigcup \mathcal{A}$, i.e., the intersection of the arrangement with the unit sphere in $W$ with respect to some norm, and the complement $M_{\mathcal{A}}=W \backslash \bigcup \mathcal{A}$. We are interested in the cohomology ring of the complement.

Combinatorial data. As mentioned in the introduction our combinatorial data is slightly extended. It is given by:

$\triangleright$ The set of all intersections of subsets of $\mathcal{A}$ partially ordered by reverse inclusion: the intersection lattice of $\mathcal{A}$, denoted by $P$. It has maximal element $T:=\bigcap \mathcal{A}$ and minimal element $\perp:=\bigcap \varnothing=W$. The lattice operation join is denoted by $\vee$ and is given in our situation by intersection.

$\triangleright P$ is furnished with a dimension function $d: P \rightarrow \mathbb{N}$ which assigns to each space its real dimension.

We assume that the following supplementary data is also given, i.e., we consider oriented arrangements.

$\triangleright$ There is a sign function

$$
\epsilon:\{(u, v) \in P \times P: u+v=W\} \longrightarrow\{ \pm 1\} .
$$

For this we assume all quotient spaces $W / u, u \in P$, to be oriented and define $\epsilon_{u, v}$ to be the degree of the linear isomorphism

$$
\left(\pi^{u \cap v, v}, \pi^{u \cap v, u}\right): W /(u \cap v) \rightarrow W / v \times W / u .
$$

For elements $u, v$ in the intersection lattice $P$ of $\mathcal{A}$ we denote by $[u, v],(u, v]$, and $[u, v)$ the respective intervals in $P$.

Generic points. The map relating combinatorial data with topological data will depend on a choice of generic points. Here by a generic point in $v, v \in P$, we simply mean a point in $v \backslash \bigcup_{z \in(v, \top]} z$. With a choice of generic points we either refer to a function $x^{u}$ for a particular $u \in P$, or to a collection $x$ of such functions $x^{u}, u \in P$,

$$
\begin{aligned}
{[W, u] } & \rightarrow W / u \\
v & \mapsto x_{v}^{u}
\end{aligned}
$$


with $x_{v}^{u} \in \pi^{u}[v] \backslash \bigcup_{v^{\prime} \in(v, u]} \pi^{u}\left[v^{\prime}\right]$.

Specific arrangements. Complex arrangements, i.e., finite families of linear subspaces of $\mathbb{C}^{n}$, will be considered as special real subspace arrangements in $\mathbb{R}^{2 n}$. In this case all of the associated quotient spaces are oriented canonically and the sign function $\epsilon$ only takes the value +1 . Therefore, for complex arrangements the orientation data is superfluous. A much larger class of subspace arrangements is given by the family of $(\geq 2)$-arrangements.

2.1. Definition. An arrangement $\mathcal{A}$ in $W$ is called a $(\geq 2)$-arrangement if $d(u)-d(v) \geq 2$ for all $u, v \in P$ with $v$ a proper subspace of $u$.

The codimension condition. As already mentioned the following notion leads to a crucial distinction when describing the ring structure of the cohomology of an arrangement.

2.2. Definition. Let $\mathcal{A}$ be a subspace arrangement in $W$, and let $u, v \in P$. We will say that $u$ and $v$ satisfy the codimension condition if they are in general position, i.e., $\operatorname{codim} u+\operatorname{codim} v=\operatorname{codim} u \cap v$, which is equivalent to $u+v=W$.

Note that this is a purely combinatorial condition as it is equivalent to the condition $d(u)+d(v)-d(u \vee v)=d(W)$.

\section{The Goresky-MacPherson isomorphism}

Given an arrangement $\mathcal{A}$ in $W$, our ultimate goal is to describe the cohomology ring of $W \backslash \bigcup \mathcal{A}$. Now, if $\epsilon>0$ is a real number and $B_{\epsilon}^{W}$, or simply $B_{\epsilon}$, denotes the corresponding open ball in $W$ (to some given norm), we have a Poincaré-Lefschetz duality isomorphism

$$
H^{k}(W \backslash \bigcup \mathcal{A}) \underset{i^{*}}{\cong} H^{k}\left(B_{\epsilon}^{W} \backslash \bigcup \mathcal{A}\right) \stackrel{\frown[W]}{\cong} H_{d(W)-k}\left(W, \bigcup \mathcal{A} \cup \mathcal{C} B_{\epsilon}^{W}\right) .
$$

Here $i$ denotes inclusion, $[W]$ the orientation class of $W$, and $\mathcal{C} B_{\epsilon}$ the complement of $B_{\epsilon}$. The isomorphism is independent of $\epsilon$ and the norm used to define $B_{\epsilon}$. To desribe the cohomology ring $H^{*}(W \backslash \bigcup \mathcal{A})$ we will work mainly in $H_{*}\left(W, \bigcup \mathcal{A} \cup \mathcal{C} B_{\epsilon}\right)$ and with the intersection product $\bullet$ there, which is defined by

$$
(\alpha \frown[W]) \bullet(\beta \frown[W])=(\alpha \smile \beta) \frown[W] .
$$

For any partially ordered set $Q$ we denote by $\Delta(Q)$ the order complex of $Q$. It is the (abstract) simplicial complex on the vertex set $Q$, whose simplices are given by chains in $Q$, i.e., sets of pairwise comparable elements. Given an intersection $u$ of $\mathcal{A}$ and generic points $x^{u}$ we define a map $\phi^{x^{u}}: \Delta[W, u] \rightarrow$ $W / u$ which is affine on simplices and satisfies

$$
\phi^{x^{u}}(w)=x_{w}^{u}, \quad w \in[W, u] .
$$

So $\phi^{x^{u}}$ is just the affine continuation of $x^{u}$. Note that we tacitly identified the abstract simplicial complex with its geometric realization and we will do so for the rest of the article. 
Now consider a simplex $\sigma=\left\langle v_{0}, \ldots, v_{k}\right\rangle \in \Delta[W, u], v_{0}<\cdots<v_{k}$. Then $\phi^{x^{u}}[\sigma] \subseteq \pi^{u}\left[v_{0}\right]$ and $\phi^{x^{u}}[\sigma] \cap \pi^{u}\left[v_{k}\right]=\left\{x_{v_{k}}^{u}\right\}$. Therefore $\phi^{x^{u}}[\Delta(W, u]] \subseteq$ $\bigcup \mathcal{A} / u$ and $\phi^{x^{u}}[\Delta[W, u)] \subseteq W / u \backslash B_{\epsilon}$ for small enough $\epsilon$. We thus have a map of pairs

$$
\phi^{x^{u}}:(\Delta[W, u], \Delta(W, u] \cup \Delta[W, u)) \rightarrow\left(W / u, \bigcup \mathcal{A} / u \cup \mathcal{C} B_{\epsilon}\right)
$$

Since we will need these pairs all the time, we set

$$
\Delta[W, u]:=(\Delta[W, u], \Delta(W, u] \cup \Delta[W, u)) .
$$

Furthermore $\left(\pi^{u}\right)^{-1}[\bigcup \mathcal{A} / u]=\bigcup \mathcal{A}_{u} \subseteq \mathcal{A}$ and $\left(\pi^{u}\right)^{-1}\left[\mathcal{C} B_{\epsilon}^{W / u}\right] \subseteq \mathcal{C} B_{\epsilon}^{W}$ when $W / u$ is given the quotient norm and we can consider the maps

$$
H_{k}(\Delta[W, u]) \stackrel{\phi_{*}^{x^{u}}}{\longrightarrow} H_{k}\left(W / u, \bigcup \mathcal{A} / u \cup \mathcal{C} B_{\epsilon}\right) \stackrel{\pi_{!}^{u}}{\longrightarrow} H_{k+d(u)}\left(W, \bigcup \mathcal{A} \cup \mathcal{C} B_{\epsilon}\right) .
$$

Here $\pi_{!}^{u}$ denotes the transfer of $\pi^{u}$, where the transfer is defined, for any map $f: M_{0}^{n_{0}} \rightarrow M_{1}^{n_{1}}$ between manifolds and $A_{i} \subseteq X_{i} \subseteq M_{i}$, such that $H^{*}\left(M_{i} \backslash A_{i}, M_{i} \backslash X_{i}\right) \stackrel{\left.\frown M_{i}\right]}{\longrightarrow} H_{*}\left(X_{i}, A_{i}\right)$ are isomorphisms and $f^{-1}\left[X_{1}\right] \subseteq X_{0}$, $f^{-1}\left[A_{1}\right] \subseteq A_{0}$, by $f_{!}: H_{k}\left(X_{1}, A_{1}\right) \stackrel{\alpha \frown\left[M_{1}\right] \mapsto f^{*} \alpha \frown\left[M_{0}\right]}{\longrightarrow} H_{k+n_{0}-n_{1}}\left(X_{0}, A_{0}\right)$, see for example [Dol72, VIII.10].

The next proposition is a formulation of the Goresky-MacPherson isomorphism [GM88], originally proven by means of stratified Morse theory. Ziegler and Živaljević [ZŽ93] gave an elementary proof based on a homotopy model for the link. We give a self-contained proof which is an adaptation of their proof. Section 8 explains the correspondence of our approach to theirs.

3.1. Proposition. Let $\mathcal{A}$ be an arrangement in $W$ and $x^{u}$ be a choice of generic points. Then the map

$$
\sum_{u \in[W, \top]} \pi_{!}^{u} \circ \phi_{*}^{x^{u}}: \bigoplus_{u \in[W, \top]} H_{*}(\Delta[W, u]) \rightarrow H_{*}\left(W, \bigcup \mathcal{A} \cup \mathcal{C} B_{\epsilon}\right)
$$

is an isomorphism.

Proof. The proof is by induction on the cardinality of the intersection lattice $P$. If the cardinality of $P$ equals 1 we must have $\mathcal{A}=\varnothing$. In this case the proposition is trivial, since $\phi^{x^{W}}$ is a homeomorphism and $\pi^{W}$ a homotopy equivalence. Now let $|P|>1$ and $a \in \mathcal{A}$ a subspace of $W$ that is not contained in any other element of $\mathcal{A}$. For any $u \in P$ we have a commutative 
diagram

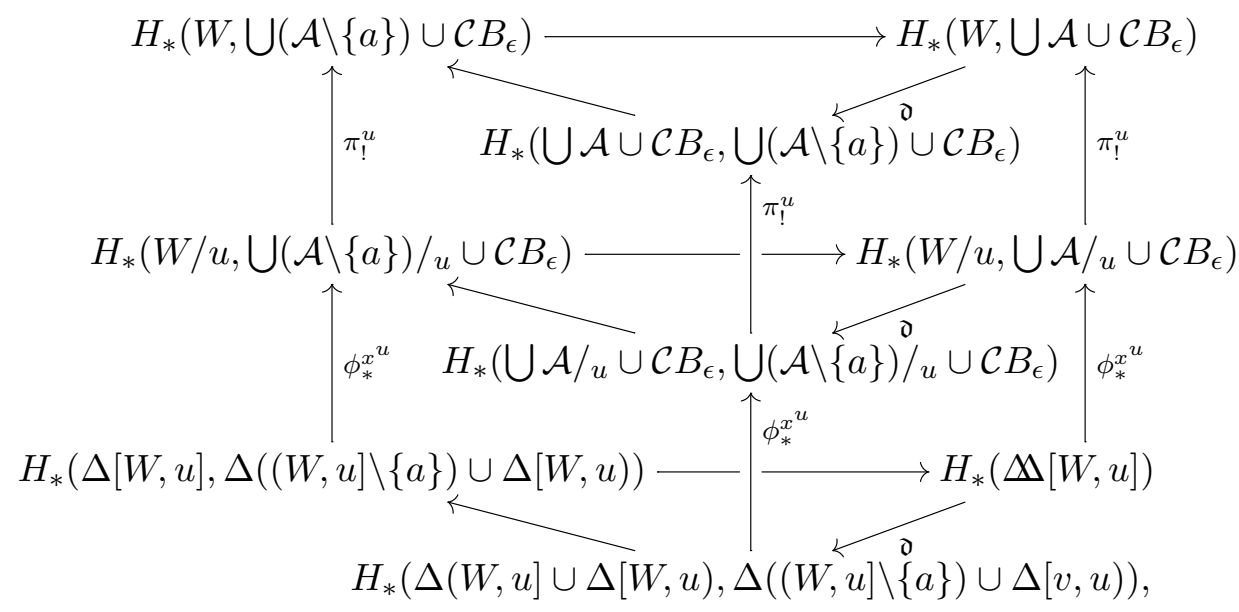

where the triangles come from triples of spaces and are exact. Our aim is to show that taking direct sums over all $u \in P$ in the lower two triangles makes the composition in the right column an isomorphism. By the five-lemma it suffices to show this for the other two columns. Regarding the left column, note that the lower group is trivial if $u=a$, and that otherwise the map $H_{*}(\Delta \backslash([W, u] \backslash\{a\})) \rightarrow H_{*}(\Delta[W, u], \Delta((W, u] \backslash\{a\}) \cup \Delta[W, u))$ induced by inclusion is an isomorphism. (Use excision and the fact that order complexes of posets containing a minimal element are contractible.) Hence, considering the arrangement in $W$ consisting of all elements of $P \backslash\{a\}$ the left column yields an isomorphism by induction. Regarding the middle column define $\mathcal{A}^{\prime}$ to be the arrangement in $a$ consisting of $\{w \cap a: w \in \mathcal{A} \backslash\{a\}\}$. Its intersection lattice is $[a, \top]$. Now for $u \in[a, \top]$ the diagram

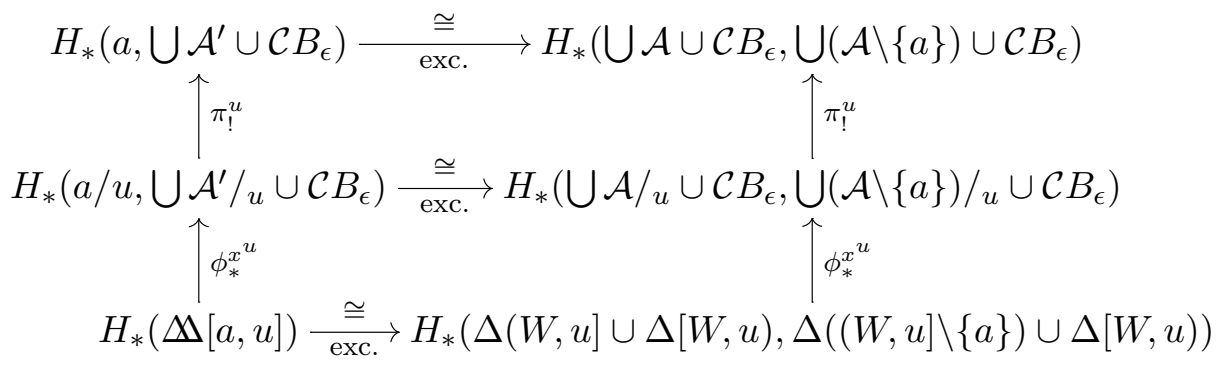

commutes, and for $u \in[W, \top], u \nsupseteq a$ the isomorphism in the bottom row also exists and shows that the group on the right is trivial. Therefore the second column of the original diagram yields an isomorphism as well.

\section{Products}

Products of lattices. Let $P$ and $Q$ be lattices. $\Delta(P \times Q)$ is just $\Delta(P) \times$ $\Delta(Q)$ endowed with the usual simplicial structure that a product of two simplicial complexes (with vertex orders) is given. Therefore, if $C_{*}$ denotes 
the ordered chain complex, there is the well known map

$$
C_{*}(\Delta P) \otimes C_{*}(\Delta Q) \stackrel{\times}{\longrightarrow} C_{*}(\Delta(P \times Q)) .
$$

It is given by

$$
\left\langle u_{0}, \ldots, u_{k}\right\rangle \otimes\left\langle v_{0}, \ldots, v_{l}\right\rangle \mapsto \sum_{\substack{0=i_{0} \leq \ldots \leq i_{k+l}=k \\ 0=j_{0} \leq \ldots \leq j_{k+1}=l \\ \forall r\left(i_{r-1}, j_{r}\right) \neq\left(i_{r}, j_{r}\right)}} \sigma_{i, j}\left\langle\left(u_{i_{0}}, v_{j_{0}}\right), \ldots,\left(u_{i_{k+l}}, v_{j_{k+l}}\right)\right\rangle
$$

for chains $u_{0}<u_{1}<\cdots<u_{k}$ and $v_{0}<v_{1}<\cdots<v_{l}$, where the $\sigma_{i, j}$ are signs determined by $\sigma_{i, j}=1$ if $k=0$ or $l=0$ and by $\mathfrak{d}(a \times b)=\mathfrak{d} a \times b+(-1)^{k} a \times \mathfrak{d} b$.

Because of the equality of pairs $\Delta P \times \Delta Q=\Delta(P \times Q)$, this induces a product

$$
\times: H_{*}(\Delta P P) \otimes H_{*}(\Delta Q) \rightarrow H_{*}(\Delta(P \times Q)) .
$$

Products of arrangements and exterior products of homology classes. Let $\mathcal{A}$ be an arrangement in $U$ with intersection lattice $P$ and $\mathcal{B}$ an arrangement in $V$ with intersection lattice $Q$. We define their product to be the arrangement in $U \times V$ given by $\mathcal{A} \times \mathcal{B}:=\{U \times v: v \in \mathcal{B}\} \cup\{u \times V: u \in \mathcal{A}\}$. Note that $(U, \bigcup \mathcal{A}) \times(V, \bigcup \mathcal{B})=(U \times V, \bigcup(\mathcal{A} \times \mathcal{B}))$. The intersection lattice of $\mathcal{A} \times \mathcal{B}$ is isomorphic to the product lattice $P \times Q$ via $P \times Q \ni(u, v) \mapsto u \times v$. We will identify them.

4.1. Proposition. Let $\mathcal{A}$ be an arrangement in $U$ with intersection lattice $P$ and $\mathcal{B}$ an arrangement in $V$ with intersection lattice $Q$. Furthermore let $u \in P, v \in Q$ and let $x^{u}, y^{v}$ be generic points. Then

$$
x^{u} \times y^{v}:[U, u] \times[V, v] \rightarrow U / u \times V / u=(U \times V) /(u \times v)
$$

is a choice of generic points for the intersection $u \times v$ in $\mathcal{A} \times \mathcal{B}$, and for $a \in H_{k}(\Delta[U, u]), b \in H_{l}(\Delta[V, v])$ we have

$$
\pi_{!}^{u}\left(\phi_{*}^{x^{u}}(a)\right) \times \pi_{!}^{v}\left(\phi_{*}^{y^{v}}(b)\right)=(-1)^{d(u)(d(V)-d(v)-l)} \pi_{!}^{u \times v}\left(\phi_{*}^{x^{u} \times y^{v}}(a \times b)\right) .
$$

Proof. The first statement is clear. For the second, note that we have $\phi^{x^{u}} \times$ $\phi^{y^{v}}=\phi^{x^{u} \times y^{v}}$ and $\pi^{u} \times \pi^{v}=\pi^{u \times v}$. Therefore the diagram

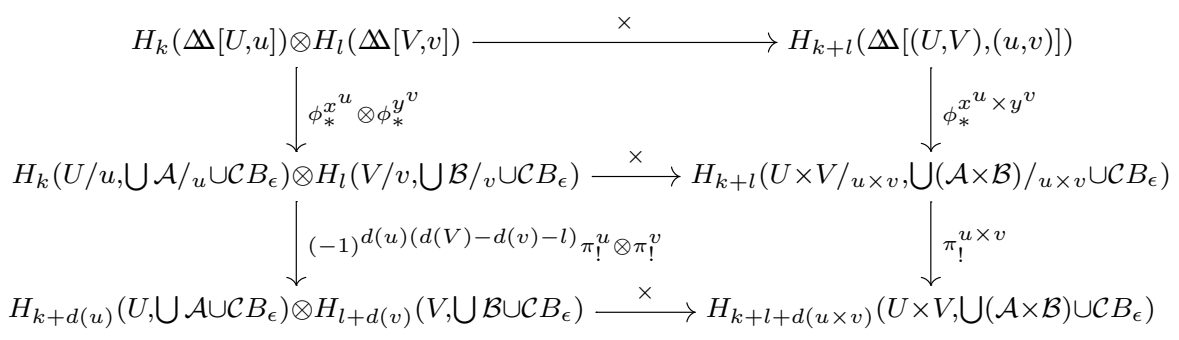

commutes. 
Intersection products of homology classes satisfying the codimension condition. Let $\mathcal{A}$ be an arrangement in $W$ and $u, v$ intersections in $\mathcal{A}$. The join operation

$$
\begin{aligned}
\vee:[W, v] \times[W, u] & \rightarrow[W, u \cap v] \\
(z, w) & \mapsto z \cap w
\end{aligned}
$$

is an isotonic map and therefore induces a simplicial map of the associated order complexes. Now assume that $u$ and $v$ satisfy the codimension condition $u+v=W$. Then $\vee$ is injective and induces a simplicial map of pairs

$$
\Delta[W, v] \times \Delta[W, u] \longrightarrow \Delta[W, u \cap v] .
$$

Furthermore, from the injectivity follows that for given generic points $x^{u}$ and $x^{v}$ there are generic points $x^{u \cap v}$ such that

$$
\left(\pi^{u \cap v, v}, \pi^{u \cap v, u}\right)\left(x_{w \cap z}^{u \cap v}\right)=\left(x_{z}^{v}, x_{w}^{u}\right) \quad \text { for all } w \in[W, u], z \in[W, v] .
$$

Here $\left(\pi^{u \cap v, v}, \pi^{u \cap v, u}\right): W /(u \cap v) \rightarrow W / v \times W / u$ is the linear isomorphism we used to define $\epsilon_{u, v}$.

4.2. Proposition. Let $\mathcal{A}$ be an arrangement in $W$ and $u, v$ intersections in $\mathcal{A}$, such that $u+v=W$. Given generic points $x^{u}$ and $x^{v}$ we have for $a \in H_{k}\left(\Perp_{\|}[W, u]\right), b \in H_{l}\left(\boldsymbol{\Perp}_{[W}[W]\right)$, and the generic points $x^{u \cap v}$ constructed above, that

$$
\pi_{!}^{u}\left(\phi_{*}^{x^{u}}(a)\right) \bullet \pi_{!}^{v}\left(\phi_{*}^{x^{v}}(b)\right)=\epsilon_{u, v}(-1)^{l(d(W)-d(u))} \pi_{!}^{u \cap v}\left(\phi_{*}^{x^{u \cap v}}\left(\vee_{*}(a \times b)\right) .\right.
$$

Proof. Denoting by $\Delta$ the diagonal map $W \rightarrow W \times W$ we have

$$
\begin{aligned}
& \pi_{!}^{u}\left(\phi_{*}^{x^{u}}(a)\right) \bullet \pi_{!}^{v}\left(\phi_{*}^{x^{v}}(b)\right)= \\
& \quad=(-1)^{(d(W)+k+d(u))(l+d(v))} \Delta_{!}\left(\pi_{!}^{v}\left(\phi_{*}^{x^{v}}(b)\right) \times \pi_{!}^{u}\left(\phi_{*}^{x^{u}}(a)\right)\right) .
\end{aligned}
$$

From Proposition 4.1 we know, that

$$
\pi_{!}^{v}\left(\phi_{*}^{x^{v}}(b)\right) \times \pi_{!}^{u}\left(\phi_{*}^{x^{u}}(a)\right)=(-1)^{d(v)(d(W)-d(u)-k)} \pi_{!}^{v \times u}\left(\phi_{*}^{x^{v} \times x^{u}}(b \times a)\right) .
$$

Now consider the diagram

$$
\begin{aligned}
& H_{k+l}(\Delta[(W, W),(v, u)]) \stackrel{\vee_{*}}{\longrightarrow} H_{k+l}(\Delta[W, u \cap v]) \\
& \downarrow \phi_{*}^{x^{v} \times x^{u}} \quad \downarrow_{*}^{x^{x^{u} v}} \\
& H_{k+l}\left(W / v \times W / u, \bigcup^{2}(\mathcal{A} / v \times \mathcal{A} / u) \cup \mathcal{C} B_{\epsilon}\right) \stackrel{\epsilon_{u, v} h_{!}}{\longrightarrow} H_{k+l}\left(W / u \cap v, \bigcup^{\downarrow} \mathcal{A} / u \cap v \cup \mathcal{C} B_{\epsilon}\right) \\
& \downarrow \pi_{!}^{v \times u}=\left(\pi^{v} \times \pi^{u}\right) ! \quad \downarrow \pi_{!}^{u \cap v} \\
& H_{k+l+d(u)+d(v)}\left(W \times \stackrel{\downarrow}{W}, \bigcup(\mathcal{A} \times \mathcal{A}) \cup \mathcal{C} B_{\epsilon}\right) \stackrel{\epsilon_{u, v} \Delta !}{\longrightarrow} H_{k+l+d(u \cap v)}\left(\underset{W}{W} \bigcup \mathcal{A} \cup \mathcal{C} B_{\epsilon}\right),
\end{aligned}
$$

where $h=\left(\pi^{u \cap v, v}, \pi^{u \cap v, u}\right)$. For $\alpha \in H^{*}\left(\left(B_{\epsilon}^{W / v} \times B_{\epsilon}^{W / u}\right) \backslash \bigcup(\mathcal{A} / v \times \mathcal{A} / u)\right)$ we have

$$
\begin{aligned}
\left(h_{*} h_{!}\right)(\alpha \frown[W / v \times W / u]) & =h_{*}\left(h^{*} \alpha \frown[W /(u \cap v)]\right)= \\
= & \alpha \frown h_{*}[W /(u \cap v)]=\epsilon_{u, v} \alpha \frown[W / v \times W / u]
\end{aligned}
$$


by definition of $\epsilon_{u, v}$ and therefore $\epsilon_{u, v} h_{!}=h_{*}^{-1}$. Since furthermore

$$
h \circ \phi^{x^{u \cap v}} \circ \vee=\phi^{x^{v} \times x^{u}}
$$

by construction of $x^{u \cap v}$, the upper square commutes. The lower square commutes, because $\left(\pi^{v} \times \pi^{u}\right) \circ \Delta=h \circ \pi^{u \cap v}$. Multiplying the signs together with $\vee_{*}(a \times b)=(-1)^{k l} \vee_{*}(b \times a)$ yields the desired result.

Intersection products of homology classes not satisfying the codimension condition. Now let $\mathcal{A}$ be an arrangement in $W$ and $u, v$ intersections in $\mathcal{A}$ with $u+v \neq W$. Then there exists a non-trivial linear functional $\Lambda: W \rightarrow \mathbb{R}$ with kernel containing $u+v$. Denote the induced functionals on $W / u$ and $W / v$ by $\Lambda_{u}$ and $\Lambda_{v}$, respectively. Now there are generic points $x^{u}$ and $y^{v}$ such that

$$
\begin{aligned}
& \Lambda_{u}\left(x_{w}^{u}\right) \geq 0 \text { for all } w \in(W, u] \text {, } \\
& \Lambda_{u}\left(x_{W}^{u}\right)>0, \\
& \Lambda_{v}\left(y_{w}^{v}\right) \leq 0 \text { for all } w \in(W, v] \text {, } \\
& \Lambda_{v}\left(y_{W}^{v}\right)<0 \text {. }
\end{aligned}
$$

4.3. Proposition. Let $\mathcal{A}$ be an arrangement in $W$ and $u, v$ intersections in $\mathcal{A}$ with $u+v \neq W$. Then, for generic points $x^{u}$ and $y^{v}$ as above, the composition

$$
\begin{aligned}
H_{*}(\Delta[W, u]) \otimes H_{*}(\Delta[W, v]) & \\
\stackrel{\left(\pi_{!}^{u} \circ \phi_{*}^{x^{u}}\right) \otimes\left(\pi_{!}^{v} \circ \phi_{*}^{y^{v}}\right)}{\longrightarrow} H_{*}\left(W, \bigcup \mathcal{A} \cup \mathcal{C} B_{\epsilon}\right) \otimes & H_{*}\left(W, \bigcup \mathcal{A} \cup \mathcal{C} B_{\epsilon}\right) \\
& \stackrel{\bullet}{\longrightarrow} H_{*}\left(W, \bigcup \mathcal{A} \cup \mathcal{C} B_{\epsilon}\right)
\end{aligned}
$$

is the zero map.

Proof. The idea is roughly that the carriers of $\operatorname{Im}\left(\pi_{!}^{u} \circ \phi_{*}^{x^{u}}\right)$ and $\operatorname{Im}\left(\pi_{!}^{v} \circ \phi_{*}^{y^{v}}\right)$ are seperated by the hyperplane $\operatorname{ker} \Lambda$, such that these carriers intersect only in $\bigcup \mathcal{A}$.

Setting $X:=\phi^{x^{u}}[[W, u]], Y:=\phi^{y^{v}}[[W, v]]$ we have

$$
X \cap \Lambda_{u}^{-1}[(-\infty, 0]] \subseteq \bigcup \mathcal{A} / u, \quad Y \cap \Lambda_{v}^{-1}[[0,+\infty)] \subseteq \bigcup \mathcal{A} / v,
$$

and therefore, with $\tilde{X}:=\left(\pi^{u}\right)^{-1}[X]$ and $\tilde{Y}:=\left(\pi^{v}\right)^{-1}[Y]$,

$$
\tilde{X} \cap \Lambda^{-1}[(-\infty, 0]] \subseteq \bigcup \mathcal{A}, \quad \tilde{Y} \cap \Lambda^{-1}[[0,+\infty)] \subseteq \bigcup \mathcal{A}
$$


It follows that $\tilde{X} \cap \tilde{Y} \subseteq \cup \mathcal{A}$ and that the lower left group in the following diagram is zero.

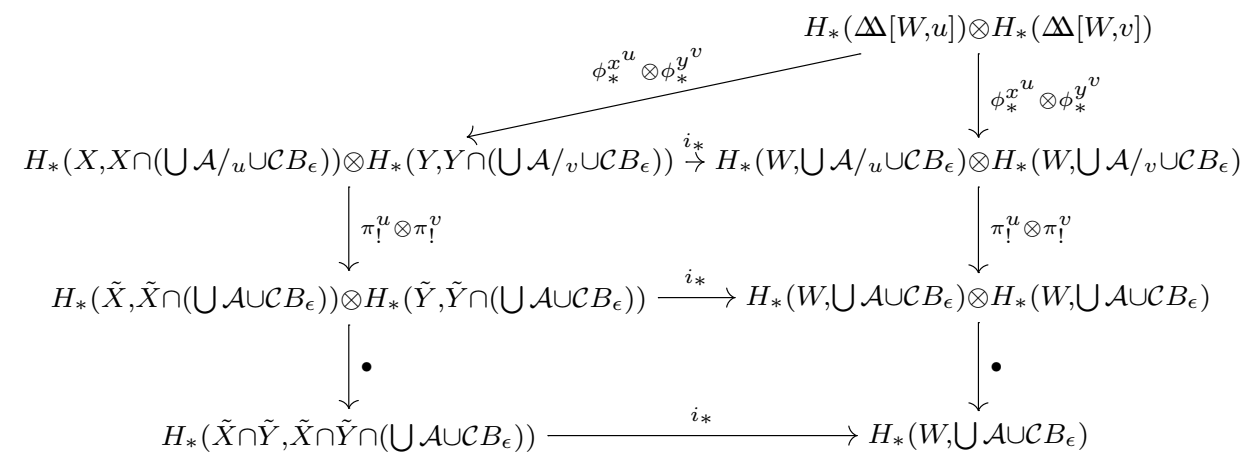

The diagram is commutative. To see this, note that if $i: W \rightarrow W$ is the identity map, and $A_{i} \subset X_{i} \subset W, i=0$, 1 , with $\left(A_{0}, X_{0}\right) \subset\left(A_{1}, X_{1}\right)$ and such that $\frown[W]: H^{*}\left(M-A_{i}, M-X_{i}\right) \rightarrow H_{*}\left(X_{i}, A_{i}\right)$ are isomorphisms, the maps $i_{*}, i_{!}: H_{*}\left(X_{0}, A_{0}\right) \rightarrow H_{*}\left(X_{1}, A_{1}\right)$ are both defined and agree.

\section{ON $(\geq 2)$-ARRANGEMENTS}

In Proposition 4.2 and Proposition 4.3 we computed the intersection product of homology classes. These were obtained for particular choices of generic points. For $(\geq 2)$-arrangements the following lemma will allow us to work with any choice of generic points.

5.1. Lemma. Let $\mathcal{A}$ be a ( $\geq 2$ )-arrangement in $W$, let $u \in P$ be an intersection of $\mathcal{A}$, and $x^{u}, \tilde{x}^{u}$ be choices of generic points for $u$. Then the affine extensions $\phi^{x^{u}}$ and $\phi^{\tilde{x}^{u}}$ are homotopic as maps of pairs $\mathbb{\Delta}[W, u] \rightarrow$ $\left(W, \bigcup \mathcal{A} / u \cup \mathcal{C} B_{\epsilon}\right)$.

Proof. For any $w \in[W, u]$ the space $\pi^{u}[w] \backslash \bigcup_{w^{\prime} \in(w, u]} \pi^{u}\left[w^{\prime}\right]$ is connected by the $(\geq 2)$-assumption. Choose a path $X_{w}$ connecting $x_{w}^{u}$ and $\tilde{x}_{w}^{u}$. Then the homotopy is given by the affine extension of $X$.

Consequently, in the situation of a $(\geq 2)$-arrangement the Goresky-MacPherson isomorphism from Proposition 3.1 is independent of the choice of generic points. In particular, we obtain a complete combinatorial description of the intersection product via the sequence of maps

$$
\begin{aligned}
H_{*}(\Delta[W, u]) \otimes H_{*}(\Delta[W, v]) & \longrightarrow H_{*}\left(W, \bigcup \mathcal{A} \cup \mathcal{C} B_{\epsilon}\right) \otimes H_{*}\left(W, \bigcup \mathcal{A} \cup \mathcal{C} B_{\epsilon}\right) \\
& \stackrel{\bullet}{\longrightarrow} H_{*}\left(W, \bigcup \mathcal{A} \cup \mathcal{C} B_{\epsilon}\right) \\
& \cong \bigoplus_{w \in[W, \top]} H_{*}(\Delta[W, w])
\end{aligned}
$$


5.2. Theorem. If $\mathcal{A}$ is a ( $\geq 2)$-arrangement, then the intersection product is given by the combinatorial data as follows.

$$
\begin{aligned}
& H_{k}(\Delta[W, u]) \otimes H_{l}(\Delta[W, v]) \longrightarrow H_{k+l}(\Delta[W, u \vee v]) \\
& a \otimes b \longmapsto\left\{\begin{array}{l}
\epsilon_{u, v}(-1)^{l(d(W)-d(u))} \vee_{*}(a \times b), \\
\text { if } d(u)+d(v)-d(u \vee v)=d(W), \\
\text { else. }
\end{array}\right.
\end{aligned}
$$

Proof. Immediate by Proposition 3.1, Proposition 4.2, and Proposition 4.3 together with Lemma 5.1.

Via duality this yields a description of the $\smile$-product multiplication of the integral cohomology of the complement of $\mathcal{A}$.

\section{Geometric $(\geq 2)$-arrangements.}

Historical remarks. In the case of a complex hyperplane arrangement a description of the integral cohomology ring in terms of generators and relations was given by Arnol'd [Arn69] and Brieskorn [Bri73]. A combinatorial description of their result was given by Orlik and Solomon [OS80].

Goresky and MacPherson [GM88, Chapter III] computed the Poincaré polynomial for the class of $c$-arrangements which can be considered as generalized hyperplane arrangements.

5.3. Definition. An arrangement $\mathcal{A}=\left\{H_{1}, \ldots, H_{m}\right\}$ in an $n$-dimensional real vector space $W$ is called a $c$-arrangement, if $\operatorname{codim}_{\mathbb{R}}\left(H_{i}\right)=c$ for all $i$, and for all pairs of elements $u \subset v$ in the intersection lattice $P$ we have that $\operatorname{codim}_{\mathbb{R}}(u \subset v)$ is an integral multiple of $c$.

Ziegler gave a presentation of the integral cohomology of the complement of 2-arrangements [Zie93] generalizing the result of Orlik and Solomon. He used this presentation to give an example of a complex and a 2-arrangement with the same combinatorial data - intersection poset and dimension function - which yield different cohomology rings. In his article the orthogonal complements of the spaces are oriented by the choice of normal vectors for pairs of real hyperplanes whose intersections yield the codimension-2-spaces in the arrangement.

Hyperplane- and $c$-arrangements belong to the much larger class of arrangements with geometric intersection lattice.

5.4. Definition. A lattice $L$ is geometric, if the following conditions hold:

$\triangleright$ it is atomic, i.e., every element is the join of a set of atoms,

$\triangleright$ it admits a rank function $\mathrm{rk}$, i.e., for all $x \in L$ all maximal chains from the smallest element to $x$ have the same length (which is $\operatorname{rk}(x)+1)$,

$\triangleright$ its rank function is semimodular, i.e., for all $x, y \in L$ we have

$$
r k(x \vee y)+\operatorname{rk}(x \wedge y) \leq \operatorname{rk}(x)+\operatorname{rk}(y) .
$$


For the class of complex arrangements with geometric intersection lattice Feichtner and Ziegler [FZ00], and Yuzvinsky [Yuz98], [Yuz99] gave a presentation of the integral cohomology ring of the complement.

A presentation for the cohomology ring of a geometric $(\geq 2)$-arrangement. We give a presentation for the cohomology ring of the complement of a $(\geq 2)$-arrangement with geometric intersection lattice, thus generalizing the results in [FZ00], [Yuz98], respectively. Since our theorem can be easily achieved by the same techniques as in Yuzvinsky's article [Yuz99] plugging in our stronger Theorem 5.2, we omit the proof. It is essentially based on the understanding of the homology of geometric lattices and the cross cut complex (cf. [Bjö94]).

Recall that a subset $\sigma$ of atoms of a geometric lattice $L$ is called independent if $\mathrm{rk} \bigvee \sigma=|\sigma|$, otherwise it is called dependent.

5.5. Theorem. Let $\mathcal{A}$ be a $(\geq 2)$-arrangement in $W$ with geometric intersection lattice L. Fix an arbitrary linear order on the set of atoms of $L$. Then the integral cohomology ring of the complement $M_{\mathcal{A}}$ has the presentation

$$
0 \longrightarrow I \longrightarrow T^{*}\left(\bigoplus_{\sigma \text { ind. }} \mathbb{Z} \cdot e_{\sigma}\right) \stackrel{\pi}{\longrightarrow} H^{*}\left(M_{\mathcal{A}} ; \mathbb{Z}\right) \longrightarrow 0
$$

where $T^{*}$ denotes the tensor algebra and the sum in the middle is over all independent sets $\sigma$ of atoms of $L$, and $\pi\left(e_{\sigma}\right) \in H^{(d(W)-d(\cap \sigma))-|\sigma|}\left(M_{\mathcal{A}} ; \mathbb{Z}\right)$. The ideal I of relations is generated by the following three families of elements.

(i) For every minimal dependent set $\sigma=\left\{a_{0}, \ldots, a_{k}\right\}$ of atoms of $L$ :

$$
\sum_{i=0}^{k}(-1)^{i} e_{\sigma \backslash\left\{a_{i}\right\}} \cdot
$$

(ii) For all pairs $\sigma, \tau$ of independent sets of atoms of $L$ such that $d(\cap \sigma)+$ $d(\bigcap \tau)-d(\bigcap \sigma \cap \bigcap \tau)=d(W):$

$$
e_{\sigma} \wedge e_{\tau}-\epsilon_{\cap \sigma, \cap \tau}(-1)^{|\tau|(d(W)-d(\cap \sigma))}(-1)^{\operatorname{sign}(\sigma, \tau)} e_{\sigma \cup \tau},
$$

where $\operatorname{sign}(\sigma, \tau)$ is the sign of the permutation that orders the elements of $\sigma$ followed by the elements of $\tau$, each already ordered, ascendingly according to the chosen linear order.

(iii) For all pairs $\sigma, \tau$ of independent sets of atoms of $L$ such that $d(\bigcap \sigma)+$ $d(\bigcap \tau)-d(\bigcap \sigma \cap \bigcap \tau) \neq d(W):$

$$
e_{\sigma} \wedge e_{\tau} .
$$

In particular we obtain a presentation for general real $c$-arrangements generalizing Ziegler's result in [Zie93].

5.6. Corollary. Let $\mathcal{A}=\left\{H_{1}, \ldots, H_{m}\right\}$ be a c-arrangement in $W$. Then the integral cohomology ring of the complement $M_{\mathcal{A}}$ has the presentation

$$
0 \longrightarrow I \longrightarrow \Lambda^{*} \mathbb{Z}^{m} \stackrel{\pi}{\longrightarrow} H^{*}\left(M_{\mathcal{A}} ; \mathbb{Z}\right) \longrightarrow 0,
$$


if $c$ is even, $\Lambda^{*}$ denoting the exterior algebra, and

$$
0 \longrightarrow I \longrightarrow S^{*} \mathbb{Z}^{m} \stackrel{\pi}{\longrightarrow} H^{*}\left(M_{\mathcal{A}} ; \mathbb{Z}\right) \longrightarrow 0,
$$

if $c$ is odd, $S^{*}$ denoting the symmetric algebra, and $\pi\left(e_{i}\right) \in H^{c-1}\left(M_{\mathcal{A}} ; \mathbb{Z}\right)$ for the canonical basis $\left\{e_{1}, \ldots, e_{m}\right\}$ of $\mathbb{Z}^{m}$. The ideal $I$ of relations is generated by

$$
\sum_{i=0}^{k}(-1)^{i} \epsilon\left(a_{0}, \ldots, \widehat{a_{i}}, \ldots, a_{k}\right) e_{a_{0}} \wedge \cdots \wedge \widehat{a_{a_{i}}} \wedge \cdots \wedge e_{a_{k}},
$$

for all minimal dependent sets $\left\{H_{a_{0}}, \ldots, H_{a_{k}}\right\}$, where

$$
\epsilon\left(i_{0}, \ldots, i_{r}\right)=\epsilon_{H_{i_{0}}, H_{i_{1}}} \epsilon_{H_{i_{0}} \cap H_{i_{1}}, H_{i_{2}}} \cdots \epsilon_{H_{i_{0}} \cap H_{i_{1}} \cap \cdots \cap H_{i_{r-1}}, H_{i_{r}}}
$$

for any subset $\left\{i_{0}, \ldots, i_{r}\right\} \subseteq\{1, \ldots, m\}$.

\subsection{Remarks.}

$\triangleright$ In the light of the Goresky-MacPherson isomorphism from Proposition 3.1 the map $\pi$ in Theorem 5.5 is given explicitly by:

$$
\begin{aligned}
\pi: \Lambda^{*}\left(\bigoplus_{\sigma \text { ind. }} \mathbb{Z} \cdot e_{\sigma}\right) & \longrightarrow \bigoplus_{u \in[W, \top]} H_{*}(\Delta \backslash[W, u]) \\
e_{\left\{a_{0}, a_{1}, \ldots, a_{k}\right\}} & \longmapsto \vee_{*}\left(\left\langle W, a_{0}\right\rangle \times\left\langle W, a_{1}\right\rangle \times \cdots \times\left\langle W, a_{k}\right\rangle\right) \\
& \in H_{k+1}\left(\Delta \backslash\left[W, a_{0} \cap a_{1} \cap \cdots \cap a_{k}\right]\right) .
\end{aligned}
$$

$\triangleright$ The sign $\epsilon\left(i_{0}, \ldots, i_{r}\right)$ in Corollary 5.6 is also given by the degree of the linear isomorphism

$$
W /\left(H_{i_{0}} \cap H_{i_{1}} \cap \cdots \cap H_{i_{r}}\right) \longrightarrow W / H_{i_{r}} \times W / H_{i_{r-1}} \times \cdots \times W / H_{i_{0}}
$$

given by the canonical projections onto the factors.

\section{Counterexamples Regarding the general CASe}

The results from the previous section fail if we are not dealing with $(\geq 2)$ arrangements.

Consider an arrangement $\mathcal{A}$ that has a pair $u, w$ of intersections with $\operatorname{codim}_{\mathbb{R}}(u \subset w)=1$. Then

$$
\pi^{u}[w] \backslash \bigcup_{w^{\prime} \in(w, u]} \pi^{u}\left[w^{\prime}\right]=\pi^{u}[w] \backslash \pi^{u}[u]=w / u \backslash u / u,
$$

which is the real line minus one point. Hence, there are up to homotopy two possible choices for $x_{w}^{u}$.

Already the arrangement in $\mathbb{R}^{3}$ given by the coordinate hyperplanes shows that the following phenomena occur.

$\triangleright$ The Goresky-MacPherson isomorphism from Proposition 3.1 depends on the choice of the generic points.

$\triangleright$ The particular choice of generic points in Proposition 4.2 is necessary. 
In the general case non-trivial muliplication can occur even if the codimension condition is not satisfied. To construct an example consider the "Hopf-arrangement" of six affine lines in $\mathbb{R}^{3}$ as shown in Figure 1.

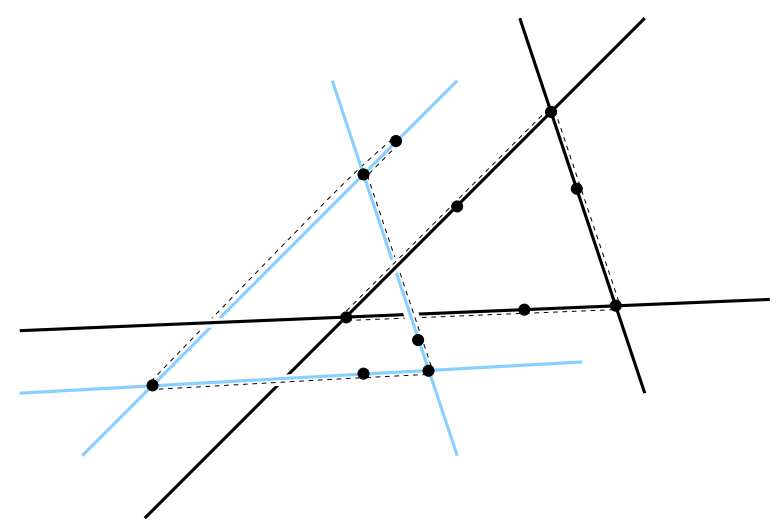

Figure 1. The "Hopf-arrangement" in $\mathbb{R}^{3}$

Let the arrangement $\mathcal{A}$ be given by the spans of $0 \in \mathbb{R}^{4}$ and the respective lines in the "Hopf-arrangement", where $\mathbb{R}^{3}$ is to be considered as the subspace $\mathbb{R}^{3} \times\{1\} \subset \mathbb{R}^{4}$. Then the order complex $\Delta\left(\left(\mathbb{R}^{4}, 0\right)\right)$ consists of two circles. A particular choice of generic points $x^{0}$ is partially illustrated in Figure 1. $x_{0}^{0}$ and $x_{\mathbb{R}^{4}}^{0}$ are not shown. They are not of interest anyway, since $x_{0}^{0}=0$ and there is only one choice up to homotopy for $x_{\mathbb{R}^{4}}^{0}$ since the complement of the arrangement is connected. The two dashed circles yield generating classes of $\tilde{H}_{1}\left(\Delta\left(\left(\mathbb{R}^{4}, 0\right)\right)\right) \cong H_{3}\left(\Delta\left[\mathbb{R}^{4}, 0\right]\right)$; Section 8 gives information on this isomorphism and how to interpret it in terms of the link $L_{\mathcal{A}}$. Since the circles are linked, the corresponding homology classes have non-trivial intersection product, although $0+0 \neq \mathbb{R}^{4}$.

In the last example one could suspect that there might have been a more clever choice of generic points to circumvent the non-trivial multiplication. This is not the case, in general it can happen that any choice of generic points leads to non-trivial multiplication in the case where the codimension condition is not satisfied. For this consider four distinct lines through the origin in $\mathbb{R}^{2}$. It is easy to see that any choice of generic points $x^{0}$ allows the choice of classes in $\tilde{H}_{0}\left(\Delta\left(\left(\mathbb{R}^{2}, 0\right)\right)\right) \cong H_{2}\left(\Delta\left[\mathbb{R}^{2}, 0\right]\right)$ that multiply nontrivially: we can assume that $x_{l}^{0} \in \mathbb{S}^{1}$ for any line $l$. Now choose two pairs of such points that are linked in the 1-sphere. The corresponding classes multiply non-trivially.

\section{A RESUlt REgARDing GENERAL ARRANGEMENTS}

The examples above showed that one cannot expect to have a theorem like Theorem 5.2 for more than $(\geq 2)$-arrangements. But even for a general real 
arrangement, the combinatorics of the arrangement describes some aspects of the product structure of its complement.

A filtration of the homology of the link. Let $\mathcal{A}$ be a real linear subspace arrangement in $W$ with intersection lattice $P$. Consider the following filtration of $H_{*}\left(W, \cup \mathcal{A} \cup \mathcal{C} B_{\epsilon}\right)$. For $u \in P$, define

$$
\begin{aligned}
F_{u} & =F_{u}\left(H_{*}\left(W, \bigcup \mathcal{A} \cup \mathcal{C} B_{\epsilon}\right)\right) \\
& =\operatorname{Im}\left(H_{*}\left(W, \bigcup \mathcal{A}_{u} \cup \mathcal{C} B_{\epsilon}\right) \stackrel{\text { incl }_{*}}{\longrightarrow} H_{*}\left(W, \bigcup \mathcal{A} \cup \mathcal{C} B_{\epsilon}\right)\right),
\end{aligned}
$$

where $\mathcal{A}_{u}=\{w \in P: u \subseteq w\}$. Note that this corresponds to the filtration $F_{u}\left(H^{*}(W \backslash \bigcup \mathcal{A})\right)=\operatorname{Im}\left(H^{*}\left(W \backslash \bigcup \mathcal{A}_{u}\right) \stackrel{\text { incl }_{*}}{\longrightarrow} H^{*}(W \backslash \bigcup \mathcal{A})\right)$ on the cohomology of the complement.

We want to use the filtration to relate the intersection product on the homology with the combinatorial product as given in Theorem 5.2. We first note that the intersection product respects the filtration.

7.1. Lemma. Let $a \in F_{u}$ and $b \in F_{v}$. Then $a \bullet b \in F_{u \cap v}$ for the intersection product in homology.

Proof. Immediate by $\bigcup A_{u} \cup \bigcup A_{v} \subseteq \bigcup A_{u \cap v}$ and the fact that the intersection product is natural with respect to inclusions.

A direct consequence is the following.

7.2. Lemma. Let $u, v \in P$ satisfy the codimension condition, i.e., $u+v=$ $W$. Assume $a \in F_{u}$ and $b \in \sum_{w \in[W, v)} F_{w}$, then $a \bullet b \in \sum_{w \in[W, u \cap v)} F_{w}$.

The associated graded ring. Associated to the filtration $\left(F_{u}\right)_{u \in P}$ are the graded abelian groups $G_{u}$ given by

$$
G_{u}=F_{u} / \sum_{w \in[W, u)} F_{w}, \quad u \in P .
$$

Consider the following induced product $\star$ on $G=\bigoplus_{u \in P} G_{u}$.

$$
\begin{aligned}
\star: G_{u} \otimes G_{v} & \rightarrow G_{u \cap v} \\
{[a] \star[b]: } & := \begin{cases}{[a \bullet b],} & \text { if } u+v=W, \\
0, & \text { else. }\end{cases}
\end{aligned}
$$

Lemma 7.1 and Lemma 7.2 show that $\star$ is well-defined.

Relation to Goresky-MacPherson isomorphisms. We can describe the filtration in terms of a Goresky-MacPherson isomorphism.

7.3. Lemma. Let $x$ be some choice of generic points. Then

$$
F_{u}\left(H_{*}\left(W, \bigcup \mathcal{A} \cup \mathcal{C} B_{\epsilon}\right)\right)=\bigoplus_{w \in[W, u]}\left(\pi_{!}^{u} \circ \phi_{*}^{x^{u}}\right)\left[H_{*}(\Delta[W, w])\right] .
$$


Proof. $\sum_{w \in[W, u]} \pi_{!}^{w} \circ \phi_{*}^{x^{w}}$ is a Goresky-MacPherson isomorphism for $\mathcal{A}_{u}$, and the commutativity of the diagram

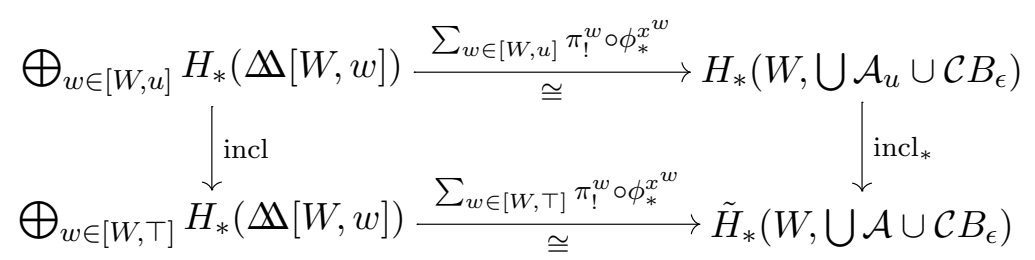

implies the lemma.

Note that although the direct sum decomposition depends on the choice of generic points, the filtration does not. The following proposition gives important information on this dependence. It describes the effect of changing generic points on the images of homology classes under the corresponding Goresky-MacPherson isomorphisms.

7.4. Proposition. Let $x^{u}$ and $\tilde{x}^{u}$ be choices of generic points for the arrangement $\mathcal{A}$, and let $a \in H_{*}(\Delta[W, u])$. Then

$$
\left(\pi_{!}^{u} \circ \phi_{*}^{x^{u}}\right)(a)-\left(\pi_{!}^{u} \circ \phi_{*}^{\tilde{x}^{u}}\right)(a) \in \sum_{w \in[W, u)} F_{w} .
$$

Proof. It is sufficient to consider choices of $x^{u}$ and $\tilde{x}^{u}$ that differ for a single $v<u$ only. Now consider the subcomplex $C$ of $\Delta[W, u]$ consisting of all simplices containing $v$. Every vertex $w$ of $C$ is comparable with $v$, that is, either contains $v$ or is contained by $v$. Therefore $x_{w}^{u} \in \bigcup \mathcal{A}_{v} / u$ for every vertex $w$ of $C, w \neq W$, and of course $\tilde{x}_{v}^{u} \in \bigcup \mathcal{A}_{v} / u$. This implies the result.

Now we only have to put things together to relate the combinatorial product on $\bigoplus_{u \in[W, T]} H_{*}(\Delta[W, u])$ as in Theorem 5.2 and the multiplication $\star$ on $G$ induced by the intersection product. Note that this will however say nothing about products of classes not satisfying the codimension condition. 7.5. Theorem. Any choice $x$ of generic points for $\mathcal{A}$ induces an isomorphism of rings

$$
\Psi: \bigoplus_{u \in[W, \top]} H_{*}(\Delta[W, u]) \rightarrow G
$$

by

$$
\begin{aligned}
H_{*}(\Delta \Delta[W, u]) & \rightarrow G_{u} \\
a & \mapsto\left[\left(\pi_{!}^{u} \circ \phi_{*}^{x^{u}}\right)(a)\right],
\end{aligned}
$$

and any two of them are equal.

Proof. The fact that

$$
\sum_{u} \pi_{!}^{u} \circ \phi_{*}^{x^{u}}: \bigoplus_{u \in[W, \top]} H_{*}(\Delta[W, u]) \rightarrow H_{*}\left(W, \bigcup \mathcal{A} \cup \mathcal{C} B_{\epsilon}\right)
$$


is an isomorphism of abelian groups together with Lemma 7.3 implies that the induced homomorphism $\Psi$ is also an isomorphism of abelian groups. Because of Proposition 7.4 it is independent of $x$. Because of this independence, Proposition 4.2 suffices to ensure that $\Psi$ is a ring homomorphism.

\section{The Homology of THE LINK}

The isomorphism

$$
\bigoplus_{u} H_{*}(\Delta[W, u]) \stackrel{\cong}{\rightrightarrows} H_{*}\left(W, \bigcup \mathcal{A} \cup \mathcal{C} B_{\epsilon}\right) \stackrel{\cong}{\rightrightarrows} H^{*}(W \backslash \bigcup \mathcal{A})
$$

we have used throughout the paper is well suited for the computation of the cohomology ring of the complement, but other closely related ones may be better known to the reader. We describe the connections between some of them in this section; this will include alternate descriptions of $H_{*}(\Delta \Delta[W, u])$.

The rest of this paper is independent of this section, it may however help in the interpretation of the picture in Section 6 .

The ordered chain complex $\left.C_{*}(\Delta)[W, u]\right)$ of the pair

$$
\Delta[W, u]=(\Delta[W, u], \Delta(W, u] \cup \Delta[W, u))
$$

is free abelian on the set of all simplices in $\Delta[W, u]$ containing both of the vertices $W$ and $u$. If $W$ and $u$ do not coincide, discarding these vertices yields a bijection, decreasing dimension by 2 , into the set of all simplices in $\Delta(W, u)$ if one allows the empty simplex in $\Delta(W, u)$. This induces an isomorphism of $C_{*}(\Delta[W, u])$ with the augmented complex $\tilde{C}_{*}(\Delta(W, u))$; remarking that $\Delta[W, W]=(\{W\}, \varnothing)$, one has

$$
H_{k}(\Delta[W, u]) \cong \begin{cases}\tilde{H}_{k-2}(\Delta(W, u)), & u>W, \\ H_{k}(\text { point }), & u=W .\end{cases}
$$

Note that $\tilde{H}_{-1}(\varnothing) \cong \mathbb{Z}$, generated by the empty simplex.

The generator of $H_{0}\left(\boldsymbol{N}_{[}[W, W]\right)$ is mapped under $\pi_{!}^{W} \circ \phi_{*}^{x^{W}}$ to the orientation class $[W]$ of $W$ in $H_{d(W)}\left(W, \cup \mathcal{A} \cup \mathcal{C} B_{\epsilon}\right)$.

For $u>W$ we will use the description of this isomorphism as the composition

$$
H_{k}(\Delta[W, u]) \underset{\cong}{\stackrel{\mathfrak{d}_{\top}}{\cong}} H_{k-1}(\Delta[W, u), \Delta(W, u)) \stackrel{\mathfrak{d}_{\perp}}{\cong} \tilde{H}_{k-2}(\Delta(W, u))
$$

of the connecting homomorphism of the homology sequence of the triple $\Delta(W, u] \subset \Delta(W, u] \cup \Delta[W, u) \subset \Delta[W, u]$ together with an excision isomorphism, and the connecting homorphism of the reduced homology sequence of the pair $\Delta(W, u) \subset \Delta[W, u)$.

Since $\phi^{x^{u}}[\Delta[W, u)] \subset \mathcal{C} B_{\epsilon}$, we can consider the composition

$$
r \circ \phi^{x^{u}}: \Delta[W, u) \rightarrow \mathbb{S}_{W / u},
$$

where $r$ denotes radial projection of $\mathcal{C} B_{\epsilon}^{W / u}$ to the unit sphere $\mathbb{S}_{W / u}$ in $W / u$. Identifying $W$ with $u \times W / u$, and therefore $\mathbb{S}_{W}$ with $\mathbb{S}_{u} * \mathbb{S}_{W / u}$, and orienting 
$u$ and $\mathbb{S}_{u}$ accordingly, we get a commutative diagram

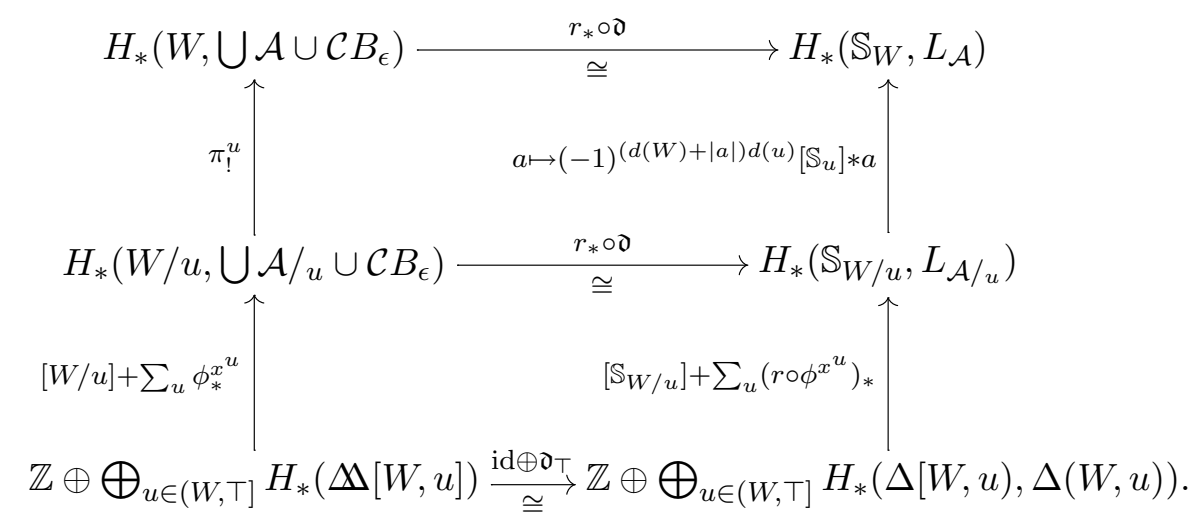

The $\mathfrak{d s}$ in the upper horizontal arrows denote compositions of connecting homomorphisms and excision isomorphisms just as for $\mathfrak{d}_{\top}$. The map $r \circ \phi^{x^{u}}$ can be considered a variant of $\phi^{x^{u}}$ using generic points in $\mathbb{S}_{W / u}$ and mapping simplices in $\Delta[W, u)$ to geodesic simplices in $\mathbb{S}_{W / u}$. We define

$$
\Phi^{x^{u}}(a):=\left[\mathbb{S}_{u}\right] *\left(r \circ \phi^{x^{u}}\right)_{*}(a)
$$

as in the right coloumn of the diagram.

If we next assume that there is a point $q \in \mathbb{S}_{W} \backslash L_{\mathcal{A}}$ such that $-x_{W}^{u}$ lies in the ray from the origin through $\pi^{u}(q)$ for every $u \in[W, \top]$, then $\Phi^{x^{u}}$ will miss $q$, and we get a commutative diagram with exact columns

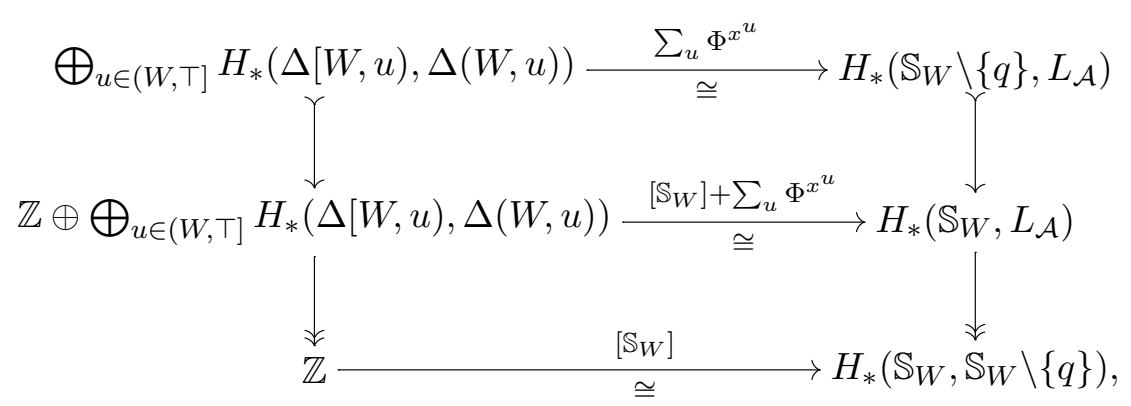

where the right column - again - fits in a long exact sequence. Now using the acyclicity of $\mathbb{S}_{W} \backslash\{q\}$ we get the diagram

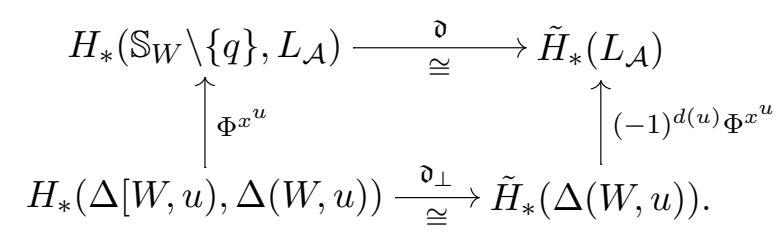


Fitting everything together gives the commutative diagram

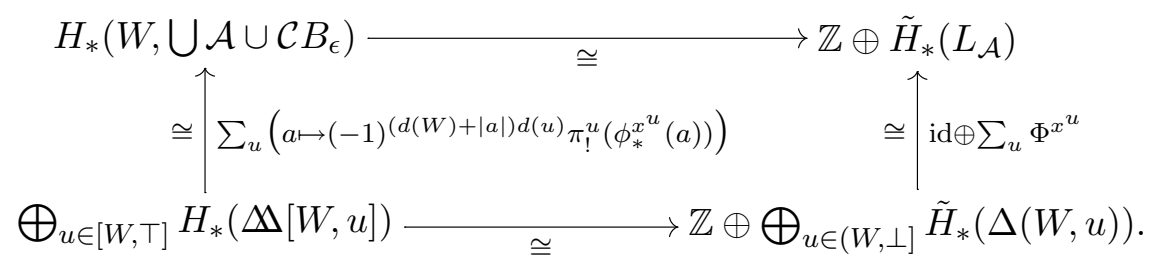

The isomorphism on the right may be easier to visualise, because the dimensions are lower. It is also the one that results from applying the functor $\tilde{H}$ to the homotopy equivalence between the link $L_{\mathcal{A}}$ and a certain quotient, identifying pairs of points, of $\coprod_{u \in(W, T]} \mathbb{S}^{d(u)-1} * \Delta(W, u)$ that is constructed in [ZŽ93]. Starting with this result and working backwards through this section gives an alternative proof of Proposition 3.1. Actually, in [ZŽ93], the $x^{u}$ are not chosen independently, but satisfy $x_{w}^{u}=\pi^{\top, u}\left(x_{u}^{\top}\right)$, but the general case would follow from this one by Lemma 7.3 and Proposition 7.4.

It is on the basis of $\bigoplus_{u \in(W, \perp]} \tilde{H}_{*}(\Delta(W, u))$ that the cohomology ring of the complement is described in [Yuz98].

\section{REFERENCES}

[Arn69] Vladimir I. Arnol'd, The cohomology ring of the colored braid group, Math. Notes 5 (1969), 138-140.

[Bjö94] Anders Björner, Topological methods, Handbook of Combinatorics (R. Graham, M. Grötschel, and L. Lovász, eds.), North Holland, Amsterdam, 1994, pp. 18191872 .

[Bri73] Egbert Brieskorn, Sur les groupes de tresses, Séminaire Bourbaki 24e année 1971/72, Lecture Notes in Mathematics 317 (1973), 21-44.

[BZ92] Anders Björner and Günter M. Ziegler, Combinatorial stratification of complex arrangements, J. Am. Math. Soc. 5 (1992), no. 1, 105-149.

[CP95] Corrado De Concini and Claudio Procesi, Wonderful models of subspace arrangements, Selecta Mathematica, New Series 1 (1995), 459-494.

[DGM99] Pierre Deligne, Mark Goresky, and Robert MacPherson, L'algèbre de cohomologie du complément, dans un espace affine, d'une famille finie de sous-espaces affines, preprint, to appear in Michigan Math. J. (1999), 23 pp.

[Dol72] Albrecht Dold, Lectures on algebraic topology, Die Grundlehren der mathematischen Wissenschaften in Einzeldarstellungen, vol. 200, Springer-Verlag, 1972.

[FZ00] Eva Maria Feichtner and Günter M. Ziegler, On cohomology algebras of complex subspace arrangements, Trans. AMS, in print (2000).

[GM88] Mark Goresky and Robert MacPherson, Stratified Morse theory, SpringerVerlag, 1988.

[Lon00] Mark de Longueville, The ring structure on the cohomology of coordinate subspace arrangements, Math. Z. 233 (2000), 553-577.

[OS80] Peter Orlik and Louis Solomon, Combinatorics and topology of complements of hyperplanes, Inventiones math. 56 (1980), 167-189.

[Yuz98] Sergey Yuzvinsky, Small rational model of subspace complement, preprint, to appear in Trans.AMS (1998), 30 pp.

[Yuz99] , Rational model of subspace complement on atomic complex, Publ. Inst. Math., Belgrade 66 (1999), no. 80, 157-164. 
[Zie93] Günter M. Ziegler, On the difference between real and complex arrangements, Math. Zeit. 212 (1993), 1-11.

[ZŽ93] Günter M. Ziegler and Rade Živaljević, Homotopy types of subspace arrangements via diagrams of spaces, Math. Ann. 295 (1993), 527-548.

Mark de Longueville, Fachbereich Mathematik, Sekr. MA 7-1, Technische Universität Berlin, Strasse des 17. Juni 136, D-10623 Berlin

E-mail address: longue@math.tu-berlin.de

Carsten A. Schultz, Institut für Mathematik II, Freie Universität Berlin, Arnimallee 2-6, D-14195 Berlin

E-mail address: cschultz@math.fu-berlin.de 